\title{
Littératie et numératie en matière de santé : facteurs clés dans la compréhension du risque de cancer
}

\author{
L Donelle, Ph.D. (1); J.F. Arocha, Ph.D. (2); L Hoffman-Goetz, Ph.D. (2)
}

\section{Résumé}

En cette ère de maladies chroniques et de prise de décision partagée, les personnes sont encouragées à participer à la prise de décisions concernant leurs soins de santé. La littératie (y compris la numératie) en matière de santé est essentielle à une participation significative, et elle est reconnue comme étant un déterminant de la santé. Cette étude visait à décrire l'influence de la littératie, soit les habiletés en lecture de textes suivis et en mathématiques, de l'angoisse des mathématiques, du niveau d'instruction et du contexte de l'information sur la capacité du participant de comprendre l'information sur la prévention du cancer colorectal diffusée sur Internet. Des données sur la littératie, la numératie et l'angoisse des mathématiques, de même que des données démographiques, ont été recueillies pour 140 Canadiens âgés de 50 ans et plus. Les participants ont obtenu des résultats satisfaisants en littératie (STOFHLA), des résultats élevés en numératie (STOFHLA), ils ont présenté des niveaux moyens de numératie en matière de santé, des niveaux faibles de numératie en général et une angoisse des mathématiques modérée. Ils avaient une meilleure compréhension de l'information courante $(9,14 / 11)$ sur le cancer colorectal comparativement à l'information peu courante $(7,64 / 11)(p<0,01)$. La littératie, la numératie, l'angoisse des mathématiques et le niveau d'instruction expliquaient $60 \%$ de la variation dans les résultats obtenus par les participants en ce qui concerne la compréhension de l'information. Il faut présenter un niveau de numératie allant d'un niveau élémentaire à un niveau avancé pour pouvoir comprendre l'information sur les risques liés au cancer diffusée sur Internet. La littératie améliore la numératie lorsque le sujet est moins bien connu. Ces constatations soulignent l'importance de présenter sur Internet de l'information en matière de santé qui tient compte de différents niveaux de littératie et de numératie.

Mots clés : communication, risque, Internet, tumeurs, littératie, numératie en matière de santé

\section{Introduction}

La littératie en matière de santé est essentielle pour comprendre l'information dans le domaine de la santé, et elle est définie comme " la mesure dans laquelle les individus sont capables d'obtenir, d'assimiler et de comprendre les renseignements et services de base en santé nécessaires à la prise de décisions appropriées dans le domaine de la santé $»^{1}$ [notre traduction]. De plus, selon l'Agence de la santé publique du Canada, la littératie est un important déterminant de la santé 2 . Pourtant, un grand nombre d'adultes affichent un faible niveau de littératie et de numératie en matière de santé. En particulier, les personnes âgées sont plus nombreuses que les jeunes à afficher un faible niveau de littératie ${ }^{3-14}$. Le pourcentage estimatif des personnes âgées présentant un niveau de littératie en santé insuffisant ou faible varie, allant de $34 \%$ chez les personnes âgées de 65 ans et plus ${ }^{4,7,14}$ à $81 \%$ chez celles qui ont 60 ans et plus ${ }^{13}$. Le faible niveau de littératie en matière de santé est considéré comme un obstacle majeur à la compréhension des diagnostics et des protocoles de traitement chez les personnes âgées ${ }^{13-15}$.

Jusqu'à récemment, la littératie en matière de santé était décrite principalement comme la capacité de comprendre la lecture, la numératie en matière de santé ne présentant que peu d'intérêt pour les chercheurs $^{16}$. La numératie en santé désigne « la mesure dans laquelle les personnes sont capables d'obtenir, d'assimiler, d'interpréter, de communiquer et d'utiliser l'information numérique, quantitative, graphique, biostatistique et probabiliste requise pour prendre des décisions éclairées en matière de santé $»^{16}$ [notre traduction]. Les rapports publiés sur la compétence en mathématiques dans le contexte des soins de santé font état de compétences insuffisantes chez les jeunes adultes $^{9,17,18}$ et les adultes plus âgés ${ }^{12,19}$. Les patients atteints de cancer qui ont un niveau de compétence faible en mathématiques seraient moins en mesure d'évaluer correctement les risques de cancer en général, de même que dans leur cas en particulier $^{10,17,20}$.

Le cancer colorectal est la quatrième cause de cancer chez les Canadiens et la deuxième principale cause de décès par cancer $^{21}$. Le nombre de nouveaux cas de cancer colorectal continuera d'augmenter en raison du vieillissement de la population $^{21}$. Bien que des programmes de dépistage du cancer colorectal aient été introduits dans certaines provinces, aucun programme national de dépistage du cancer colorectal n'a encore été mis en œuvre au Canada. Par conséquent, on encourage l'éducation en matière de santé

Coordonnées des auteurs

1 Faculté des sciences de la santé, Université de Western Ontario, London (Ontario)

2 Département d'études en santé et gérontologie, Faculté des sciences appliquées en santé, Université de Waterloo, Waterloo (Ontario)

Correspondance : Laurie Hoffman-Goetz, Département d'études en santé et gérontologie, Faculté des sciences appliquées en santé, Université de Waterloo, 200 University Avenue West, Waterloo (Ontario) N2L 3G1, Téléc. : 519746 2510, Courriel : Ihgoetz@healthy.uwaterloo.ca 
et la vigilance des patients concernant la sensibilisation au risque et le dépistage préventif. La capacité de comprendre l'information sur le cancer est une compétence essentielle dans le domaine des soins de santé; elle permet aux patients d'engager un véritable dialogue avec les fournisseurs de soins en vue d'évaluer leur propre risque de maladie et de convenir des pratiques les plus pertinentes selon ce risque $\mathrm{e}^{23,24}$. La prise de décision partagée dans le cadre $\mathrm{du}$ traitement du cancer acquiert une importance toute particulière lorsqu'un grand nombre d'options thérapeutiques sont offertes, et lorsqu'il faut évaluer différents avantages et risques dans un contexte d'incertitude ${ }^{25}$. En tant que composante de la prise de décision, la compréhension du risque met en jeu la capacité d'évaluer la gravité des préjudices potentiels qui, elle, dépend de la compréhension du message relatif à la santé et des estimations chiffrées du risque ${ }^{26}$.

Le niveau d'angoisse des mathématiques ${ }^{27}$ et le niveau d'instruction des participants sont d'autres facteurs qui influent généralement sur le niveau de numératie. La capacité de comprendre le risque dépend également de l'expérience passée et de la connaissance du sujet ${ }^{8,28-30}$. Elle peut en outre dépendre du degré de connaissance des termes et des concepts associés au risque qui constituent un vocabulaire spécialisé $^{31-32}$. Les différentes expériences dans le domaine des soins de santé peuvent également fournir à la personne un contexte qui l'aidera à mieux comprendre le sens du message lié aux soins de santé.

Étant donné que les Canadiens utilisent de plus en plus Internet comme point d'accès à l'information dans le domaine de la santé22 ${ }^{2}$ la présente étude visait à déterminer l'influence de la littératie (compréhension des textes suivis), de la numératie - en général et dans le contexte de la santé -, de l'angoisse des mathématiques, du niveau d'instruction et de la connaissance du sujet sur la capacité des adultes âgés de comprendre l'information sur le risque de cancer diffusée sur Internet.

\section{Méthodologie}

\section{Participants et procédures}

Un échantillon de commodité composé de 140 adultes âgés a été recruté dans des collectivités du Sud de l'Ontario. Pour pouvoir participer à l'étude, les personnes devaient remplir les conditions suivantes : 1) avoir entre 50 et 90 ans, 2) résider de façon autonome dans la collectivité, et 3) pouvoir lire et comprendre l'anglais. Les personnes ont participé à l'étude sur une base volontaire après avoir été informées qu'elles devraient lire de l'information en anglais sur des questions liées à la santé. Les participants qui avaient reçu un diagnostic de cancer ont été exclus. Les participants ont été recrutés par le biais d'avis affichés dans les bibliothèques publiques régionales et les centres communautaires pour personnes âgées, et d'annonces dans les journaux locaux. Les candidats admissibles ont été invités à participer à une entrevue individuelle d'une durée estimative de 60 à 90 minutes. Des services de transport public étaient disponibles à chaque lieu d'entrevue et tous les lieux étaient accessibles en fauteuil roulant. La première partie de l'entrevue a consisté à obtenir des données démographiques auprès des participants, de même qu'à évaluer leur littératie fonctionnelle en matière de santé 33 , leur numératie en général $^{10}$, leur numératie en matière de santé ${ }^{9}$ et leur angoisse des mathématiques ${ }^{34}$. La deuxième partie de l'entrevue visait à évaluer la compréhension de l'information sur le cancer colorectal diffusée sur Internet. Les participants ont reçu une somme de 40 \$ en guise de remboursement de divers frais.

La littératie fonctionnelle en matière de santé a été évaluée au moyen du Short Test of Functional Health Literacy for Adults $(\text { STOFHLA })^{33}$. Cette évaluation comprend 36 questions à choix multiples pour le volet littératie, et 4 questions pour le volet numératie. Les bonnes réponses à chacune des questions pour les volets littératie et numératie valent 2 et 7 points respectivement, pour un nombre maximal de 72 points pour le volet littératie, et de 28 points pour le volet numératie. Un résultat de 0 à 55 révèle une littératie fonctionnelle insuffisante en matière de santé; les personnes ayant un tel résultat ont souvent de la difficulté à comprendre des documents très simples. Un résultat de 56 à 66 révèle un faible niveau de littératie en matière de santé, et un résultat de 67 à 100, un niveau de littératie adéquat ${ }^{33}$. Le STOFHLA a un indice de cohérence interne, de fiabilité (alpha de Cronbach $=0,98$ ) et de validité satisfaisant comparativement au TOFHLA $\left(\mathrm{r}_{\mathrm{s}}=0,91\right)$ et au Rapid Estimate of Adult Literacy in Medicine (REALM) $\left(r_{s}=0,80\right)^{33}$.

La numératie en général a été évaluée au moyen d'un questionnaire comprenant trois questions mathématiques générales. Cet instrument évalue la notion de probabilité chez la personne, de même que la capacité de cette dernière de convertir un pourcentage en proportion, et vice versa ${ }^{10,12}$. Les résultats varient entre 0 et 3 . L'indice de cohérence interne de cet instrument est satisfaisant ${ }^{9}$. Les résultats obtenus à l'aide de cet indice de la numératie concordent avec les résultats quantitatifs relatifs à la littérature de la National Adult Literacy Survey (NALS) ${ }^{35}$.

La numératie en matière de santé a été évaluée au moyen d'un questionnaire comprenant huit questions ${ }^{9}$. L'échelle mesure la capacité des participants de distinguer les différences dans l'ampleur des risques pour la santé et d'effectuer des opérations mathématiques utilisant des pourcentages et des proportions dans le contexte de la santé9. Les participants obtenaient 1 point par bonne réponse; les résultats variaient donc entre 0 et 8 . La cohérence interne était satisfaisante (alpha de Chronbach $=0,74,0,70$ et 0,75$)^{9}$.

Enfin, l'angoisse des mathématiques a été évaluée au moyen de l'Abbreviated Math Anxiety Scale (AMAS), un questionnaire comprenant neuf questions et dont la cohérence interne (alpha de Cronbach = $0,90)$ et la fiabilité de test retest $(r=0,85)$ étaient très satisfaisantes ${ }^{36}$. Les résultats varient de 1 (niveau d'angoisse faible) à 5 (niveau d'angoisse élevé), pour une note maximale de 45 au test. Dans ce test, les participants doivent indiquer leur niveau d'angoisse dans le contexte d'opérations mathématiques ${ }^{36}$. 
La dernière partie de l'entrevue était consacrée à l'évaluation de la compréhension du risque. Les participants ont lu sur Internet deux articles distincts sur la prévention du cancer colorectal, tirés du site Web de la Société canadienne du cancer (SCC) et ciblant les consommateurs. Ces articles ont été choisis pour leur ressemblance quant au type de cancer, à la taille de la police et à la lisibilité. La sélection des articles affichés sur Internet reposait sur les critères suivants : 1) $\geq 6$;2) données numériques, présentées sous forme chiffrée ou narrative; 3 ) niveau de lecture correspondant à celui de la $12^{\mathrm{e}}$ année au plus (déterminé au moyen de l'outil d'évaluation de la lisibilité SMOG) ${ }^{37}$; et 4) longueur maximale de trois pages.

Les chercheurs et les organismes communautaires partenaires en prévention du cancer ont examiné les articles Internet admissibles en vue de déterminer l'information considérée comme « courante » ou « peu courante », et un article a été retenu pour représenter chacune de ces catégories. L'information courante comprenait des renseignements grandement publicisés, facilement accessibles, et qui reprenaient les renseignements de base fournis par la SCC pour tous les types de cancer. Pour les personnes qui cherchent de l'information sur Internet, cette page était l'un des premiers liens d'une liste concernant le cancer colorectal, et le score de lisibilité SMOG correspondait à la $10^{\mathrm{e}}$ année.

Le deuxième article Internet traitait d'un aspect moins courant du cancer colorectal : la génétique. Le score de lisibilité SMOG de cet article de 1,25 page, correspondait à la $11^{\mathrm{e}}$ ou à la $12^{\mathrm{e}}$ année. Malgré la sensibilisation accrue à l'influence de l'hérédité sur la maladie, la compréhension de la génétique par la population est généralement limitée ${ }^{38-40}$. L'article Internet de la SCC sur le cancer colorectal et la génétique a donc été choisi pour représenter l'information considérée comme peu courante pour la population en général. Les partenaires communautaires en éducation et en prévention du cancer ont collaboré à la sélection de l'article Internet.

L'article sur Internet a été imprimé sur une feuille $81 / 2$ par 11 , police de caractères 14. Des versions imprimées ont été utilisées afin d'éviter l'effet confusionnel potentiel des divers niveaux de compétence en informatique. Vu que les sites Internet sont souvent mis à jour, les pages imprimées ont permis d'assurer la diffusion d'une information uniforme du début à la fin de l'étude.

Des questions à choix multiples portant sur la littératie et la numératie, basées sur le contenu de l'article Internet, ont été utilisées pour évaluer la compréhension de l'information sur le risque. Le score de lisibilité des questions de compréhension portant sur la littératie et la numératie correspondait à la $8^{e}$ ou à la $9^{\mathrm{e}}$ année. Les questions de compréhension visaient à évaluer la compréhension du message relatif à la santé en fonction de la littératie en matière de santé (p. ex., "Que signifie incidence? »), ainsi que de la numératie en matière de santé (p. ex., "Quel est le pourcentage des hommes qui sont décédés du cancer colorectal? »). L'équipe de recherche a examiné les questions du test de compréhension (contribuant à la validité apparente et à la validité de contenu), et les questions ont fait l'objet d'un essai pilote auprès de 30 adultes âgés, dont les commentaires ont été utilisés dans le cadre de la révision du questionnaire. Les participants pouvaient utiliser librement les articles Internet imprimés pour répondre aux questions de compréhension. La présentation simultanée des deux articles Internet permettait aux participants de choisir celle qu'ils préféraient lire en premier.

\section{Analyse statistique}

Toutes les analyses ont été effectuées au moyen du progiciel SPSS, version 14.0 (SPSS, 2005). Les statistiques descriptives et les résultats obtenus par les participants pour la littératie fonctionnelle en matière de santé (STOFHLA), la numératie et l'angoisse des mathématiques ont été résumées. En se servant de l'analyse de régression multiple, les auteurs ont tenu compte des variables réponses suivantes: 1) résultats totaux pour la compréhension du risque, 2 ) résultats pour la compréhension du risque par suite de la lecture de l'article Internet courant, et 3) résultats pour la compréhension du risque par suite de la lecture de l'article Internet peu courant. Choisies a priori, les variables explicatives comprenaient la littératie fonctionnelle en matière de santé (STOFHLA), la numératie en général, la numératie en matière de santé, l'angoisse des mathématiques et le niveau d'instruction. Les variables explicatives de l'âge, des compétences auto-évaluées en anglais, de la fréquence de lecture, de la compréhension auto-évaluée des statistiques et du revenu ont été incluses dans les modèles de régression subséquents. La variable du sexe a été maintenue dans toutes les équations de régression, indépendamment de la signification statistique de cette variable.

Le test non paramétrique de Wilcoxon pour observations appariées a été utilisé pour déterminer les différences entre les résultats selon qu'ils correspondaient à l'article Internet courant ou à l'article peu courant. Le test de Mann-Whitney U a permis de déterminer les différences dans les résultats selon le sexe. Dans toutes les analyses, une valeur $p$ de 0,05 a été utilisée pour indiquer une différence qui ne pouvait vraisemblablement être attribuable au seul fait du hasard.

\section{Résultats}

\section{Caractéristiques démographiques des participants}

Les participants avaient entre 50 et 90 ans, $65 \%$ d'entre eux ayant entre 50 et 69 ans. Le nombre de femmes était plus élevé ( $\mathrm{n}=103,73,6 \%$ ) que celui des hommes ( $\mathrm{n}=37,26,4 \%$ ), et la majorité des participants étaient à la retraite $(63,6 \%)$, étaient instruits $(52,9 \%$ détenaient un diplôme d'études collégiales ou universitaires), et leur revenu annuel était faible ( < 35000 \$; 56,5 \%). La plupart des participants ( $n=102,72,9 \%$ ) possédaient un ordinateur et avaient accès à Internet. Des caractéristiques additionnelles de cet échantillon de commodité sont décrites dans un autre article ${ }^{41}$.

\section{Profils de la littératie et de la numératie}

Le tableau 1 présente la gamme des résultats concernant les niveaux de littératie en matière de santé (compréhension de textes suivis), de numératie et de l'angoisse 
des mathématiques. Il n'y avait pas de différences significatives dans les résultats totaux obtenus au STOFHLA par les hommes, d'une part, et les femmes, d'autre part (Mann-Whitney $=1797,5, p=0,52$ ), dans les résultats au STOFHLA concernant la littératie (Mann-Whitney $=1878,5, p=$ 0,89 ), ou dans les résultats au STOFHLA concernant la numératie (Mann-Whitney = $1819,5, p=0,57)$. L'angoisse des mathématiques était moins courante chez les hommes $(\overline{\mathrm{x}}=20,02$, IC à $95 \%=17,71$, $22,35)$ que chez les femmes $(\overline{\mathrm{x}}=25,09$, IC à $95 \%=23,41,26,77)$ (Mann-Whitney = $1136,50, p=0,001)$.

\section{TABLEAU 1}

\section{Résultats de l'évaluation de la littératie, de la numératie et de l'angoisse des mathématiques}

\begin{tabular}{|c|c|}
\hline Variable & $\begin{array}{l}\text { Résultats moyens } \\
\text { totaux Intervalle de } \\
\text { confiance à } 95 \% \text { ) }\end{array}$ \\
\hline Littératie STOFHLA & $\begin{array}{l}63,5 / 72 \\
(61,54,65,45)\end{array}$ \\
\hline Numératie STOFHLA & $\begin{array}{l}26,20 / 28 \\
(25,58,26,81)\end{array}$ \\
\hline $\begin{array}{l}\text { Numératie en } \\
\text { matière de santé }\end{array}$ & $\begin{array}{l}5,9 / 8 \\
(5,54,6,20)\end{array}$ \\
\hline $\begin{array}{l}\text { Angoisse des } \\
\text { mathématiques }\end{array}$ & $\begin{array}{l}23,8 / 45 \\
(22,37,25,21)\end{array}$ \\
\hline Total STOFHLA & $\begin{array}{l}89,7 / 100 \\
(87,47,91,93)\end{array}$ \\
\hline Variable & Total $\%$ \\
\hline Niveau STOFHLA & $\begin{array}{l}\text { Insuffisant = } 2 \\
\text { Faible }=7 \\
\text { Suffisant }=91\end{array}$ \\
\hline Numératie en général & $\begin{aligned} 0-1 \text { correct } & =55 \\
2 \text { correct } & =29 \\
3 \text { correct } & =16\end{aligned}$ \\
\hline
\end{tabular}
niveau de littératie en matière de santé selon le STOFHLA, du niveau de numératie en général, du niveau de numératie en matière de santé, de l'angoisse des mathématiques et du niveau d'instruction (ensemble des variables explicatives $a$ priori) aux résultats totaux pour la compréhension du risque, on a réalisé une analyse de régression multiple (voir le tableau 2). Une fois la variable du sexe prise en compte, environ $60 \%$ de la variation dans la compréhension du risque chez les participants (résultats totaux) pouvait être attribuée à l'ensemble des variables explicatives a priori suivantes : niveau de littératie selon le STOFHLA, niveau de numératie selon le STOFHLA, niveau de numératie en général, niveau de numératie en matière de santé, angoisse des mathématiques et niveau d'instruction $\left(\mathrm{F}=27,21, \mathrm{dl}=7, p<0,01, \mathrm{R}^{2}=0,598\right)$. D'autres modèles tenant compte des variables explicatives du revenu, des compétences auto-évaluées en anglais, de la fréquence de lecture et de la compréhension auto-évaluée des statistiques n’ont pas été utilisés dans le modèle final. Le modèle de régression final, le plus " parcimonieux », incluait le niveau de littératie selon le STOFHLA, le niveau de numératie selon le STOFHLA, le niveau de numératie en matière de santé et l'âge du participant, et il expliquait $57 \%$ de la variation dans les résultats totaux pour la compréhension du risque chez les participants ( $F=35,244$, $\left.\mathrm{dl}=5, p<0,01, \mathrm{R}^{2}=0,568\right)$.

Des modèles de régression additionnels ont utilisé les résultats relatifs à la compréhension de l'information courante et peu courante sur le risque en tant que variablesréponses distinctes. Une fois la variable du sexe prise en compte, $38 \%$ de la variation dans la compréhension de l'information courante sur le risque diffusée sur Internet pouvait être attribuée à l'ensemble des variables explicatives a priori $(\mathrm{F}=11,08$, $\left.\mathrm{dl}=7, p<0,01, \mathrm{R}^{2}=0,377\right)$. D'autres modèles ont révélé que le niveau de numératie selon le STOFHLA, le niveau de numératie en matière de santé et l'âge du participant ont produit le modèle de régression le plus simple $(\mathrm{F}=18,486$, $\left.\mathrm{dl}=4, p<0,01, \mathrm{R}^{2}=0,354\right)$. Aucune autre variable explicative n'a contribué d'une manière significative au modèle de régression.

L'ensemble des variables explicatives $a$ priori expliquaient $56 \%$ de la variation 
dans la compréhension de l'information peu courante sur le risque lié au cancer colorectal diffusée sur Internet $(F=23,53$, $\left.\mathrm{dl}=7, p<0,01, \mathrm{R}^{2}=0,562\right)$. Le modèle le plus parcimonieux incluait le niveau de littératie selon le STOFHLA, le niveau de numératie en général, le niveau de numératie en matière de santé, il tenait compte du sexe $(\mathrm{F}=34,675$, dl $=4, p<$ $\left.0,01, R^{2}=0,507\right)$. Aucune autre variable explicative n'a contribué d'une manière significative au modèle de régression.

Les niveaux de littératie et de numératie expliquent presque $60 \%$ de la variation dans les résultats totaux et les résultats concernant la compréhension de l'information peu courante sur le risque de cancer. Seulement $35 \%$ de la variation dans la compréhension de l'information courante sur le risque lié au cancer colorectal peut être attribuée au niveau de numératie (le niveau de littératie n'était pas une variable prédictive significative).

\section{Analyse}

Tout en reconnaissant l'influence des caractéristiques du patient (c.-à-d. soutien social, état de santé) ${ }^{26,42}$ et de la présentation (c.-à-d. formulation en termes de gain ou de perte, graphique ou texte) $)^{31,43,44}$ sur le niveau de compréhension du risque, cette étude était axée sur l'influence des niveaux de littératie et de numératie, de l'angoisse des mathématiques, du niveau d'instruction et du contexte de l'information sur la capacité des Canadiens âgés de comprendre l'information sur la prévention du cancer colorectal diffusée sur Internet. Bien que les auteurs aient conclu que la compréhension du risque était satisfaisante chez les adultes âgés ayant participé à l'étude, et que ces derniers comprenaient mieux l'information courante que l'information peu courante sur la prévention du cancer, les principaux résultats de l'étude concernent l'influence du niveau de numératie en matière de santé.

À notre connaissance, aucune autre étude publiée n'a évalué, au moyen de l'outil d'évaluation de la numératie en matière de santé, la compréhension du risque d'après l'information sur la prévention du cancer colorectal diffusée sur Internet. Un faible niveau de numératie en général a été associé à une capacité réduite à évaluer le risque de cancer en général et le risque pour le participant lui-même $e^{10,11,18,20}$. Même

si le niveau de numératie en matière de santé était un prédicteur de la capacité de comprendre tant l'information courante que l'information peu courante sur le

\section{Modèle de régression multiple pour la compréhension de l'information sur le cancer colorectal diffusée sur Internet}

\begin{tabular}{|c|c|c|c|}
\hline Variable explicative & $\begin{array}{c}\begin{array}{c}\text { Information courante } \\
\text { sur Internet }\end{array} \\
\beta \text { (IC à } 95 \%)\end{array}$ & $\begin{array}{c}\begin{array}{c}\text { Information peu } \\
\text { courante sur Internet }\end{array} \\
\beta \text { (IC à } 95 \%)\end{array}$ & $\begin{array}{c}\text { Information courante } \\
\text { et information peu } \\
\text { courante combinées } \\
\beta \text { (IC à } 95 \%)\end{array}$ \\
\hline Sexe & $0,118(-0,44,0,67)$ & $0,30(-0,36,0,96)$ & $0,00(-0,92,0,92)$ \\
\hline Âge & $-0,26(-0,53,-0,006)^{*}$ & $-0,32(-0,65,0,009)$ & $-0,678(-1,14,-0,21)^{*}$ \\
\hline Littératie STOFHLA & $0,014(-0,01,0,04)$ & $0,07,(0,04,0,09)^{* *}$ & $0,067(0,03,0,11)^{* *}$ \\
\hline Numératie STOFHLA & $0,087(0,02,0,16)^{* *}$ & $0,043(-0,04,0,13)$ & $0,10(-0,017,0,218)$ \\
\hline Numératie en général & $-0,13(-0,39,0,14)$ & $0,58(0,28,0,89)^{* *}$ & $0,466(-0,002,0,89)$ \\
\hline $\begin{array}{l}\text { Numératie en matière } \\
\text { de santé }\end{array}$ & $0,401(0,26,0,54)^{* *}$ & $0,38(0,20,0,56)^{* *}$ & $0,838(0,60,1,07)^{* *}$ \\
\hline Angoisse des mathématiques & $-0,004(-0,35,0,028)$ & $-0,006(-0,4,0,031)$ & $-0,01(-0,06,0,04)$ \\
\hline Niveau d'instruction & $0,15(-0,12,0,43)$ & $0,21(-0,11,0,53)$ & $0.363(-0.09,0.82)$ \\
\hline Modèle global & $\mathbf{F}$ & dl & $\mathbf{R}^{2}$ \\
\hline \pm Total - inf. combinée & 27,21 & 7 & $0,598^{* *}$ \\
\hline 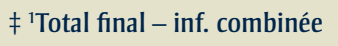 & 35,24 & 5 & $0,568^{* *}$ \\
\hline \pm Inf. courante & 11,08 & 7 & $0,377^{* *}$ \\
\hline$\ddagger^{2}$ Final - inf. courante & 18,49 & 4 & $0,354^{* *}$ \\
\hline \pm Inf. peu courante & 23,45 & 7 & $0,562^{* *}$ \\
\hline$\ddagger^{3}$ Final - inf. peu courante & 34,675 & 4 & $0,507^{* *}$ \\
\hline
\end{tabular}

Note:

$\beta=$ Coefficient bêta, IC = intervalle de confiance.

* Significatif à $p<0,05$.

** Significatif à $p<0,01$.

\pm Variables du modèle : littératie STOFHLA, numératie STOFHLA, numératie en général, numératie en matière de santé, angoisse des mathématiques et niveau d'instruction.

$\ddagger^{11}$ Modèle final parcimonieux = littératie et numératie STOFHLA, numératie en matière de santé, âge.

$\ddagger^{2}$ Modèle final parcimonieux $=$ numératie STOFHLA, numératie en matière de santé, âge.

$\ddagger^{3}$ Modèle final parcimonieux = littératie STOFHLA, numératie en général, numératie en matière de santé (le sexe a été pris en compte dans tous les modèles). 
cancer colorectal diffusée sur Internet, le niveau de numératie de base (STOFHLA) était seulement un prédicteur de la compréhension de l'information courante sur la prévention diffusée sur Internet. Par contraste, le niveau de numératie en général est un prédicteur de la compréhension de l'information plus complexe ou peu courante, mais non de la compréhension de l'information courante sur le risque de cancer colorectal.

Pour démontrer leur compréhension de l'information courante diffusée sur Internet, les participants devaient repérer, par exemple, la valeur qui désigne "le risque de décès par cancer colorectal chez les hommes " (c.-à-d., 1 sur 14). L'outil d'évaluation de la numératie STOFHLA semble être celui qui concorde le mieux avec le niveau de numératie le plus élémentaire qui consiste à identifier le chiffre ${ }^{41}$. Or, les participants qui ont répondu aux questions d'évaluation de la compréhension de l'information courante diffusée sur Internet devaient également avoir un niveau de numératie plus élevé que celui exigé par l'outil STOFHLA, p. ex., pour calculer le pourcentage d'hommes décédés du cancer colorectal, niveau de compétence caractéristique des niveaux avancés du système d'évaluation de la numératie en matière de santé ${ }^{16,45}$. Bien que l'information peu courante diffusée sur Internet ait également fait appel aux compétences en numératie de niveau élémentaire et avancé des participants, le niveau de numératie selon le STOFHLA n'était pas une variable prédictive significative. Compte tenu du manque de connaissances de la population générale concernant l'influence de la génétique sur le cancer colorectal ${ }^{39,40}$, un niveau élémentaire n'était peut-être pas suffisant $^{30}$. Les données établies ont démontré que l'étendue du vocabulaire et la connaissance du domaine contribuent à accroître la compréhension de l'information ${ }^{46}$. La connaissance du sujet améliore également la capacité de la personne de comprendre l'information sur le ris-

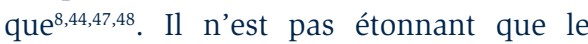
niveau de littératie en matière de santé ait aidé à comprendre uniquement l'information peu courante sur le cancer colorectal. Les mots et expressions utilisés dans l'information liée à la génétique nécessitaient peut-être la connaissance d'un vocabulaire distinct de celui requis pour comprendre l'article Internet courant. Par conséquent, les personnes qui préparent les messages axés sur la promotion de la santé voudront peut-être répéter les mots moins courants et fournir des exemples des concepts clés ${ }^{44}$. Les capacités interactives attribuables aux sources en ligne sur la santé constituent un excellent moyen d'intégrer de tels appuis à la compréhension.

Comme on s'y attendait, l'âge avancé était un facteur prédictif de résultats plus faibles en ce qui concerne la compréhension de l'information courante et de l'information peu courante combinées dans le cadre de l'évaluation du risque et pour la compréhension de l'information courante sur le risque mais non de l'information peu courante sur le risque. Une évaluation récente de la compréhension de l'information sur le cancer colorectal diffusée sur Internet chez les adultes âgés a également révélé une compréhension limitée du message ${ }^{49}$. Le lien entre l'âge plus élevé et un niveau de littératie et de numératie plus faible a déjà été établii ${ }^{12,13,33}$. Les résultats actuels sont conformes à ceux d'analyses internationales des niveaux de littératie et de numératie chez les adultes. En fait, il existe une relation inverse entre l'âge et le niveau de littératie, même après avoir pris en compte le niveau d'instruction ${ }^{50}$. L'Enquête internationale sur l'alphabétisation des adultes (EIAA) mentionne que le niveau d'instruction n'est pas un prédicteur fiable du niveau de littératie ${ }^{51}$. Les auteurs ont également constaté que le niveau d'instruction n'est pas un prédicteur significatif du niveau de littératie dans la présente étude. Bien que le niveau d'instruction joue un rôle clé dans l'acquisition des compétences en littératie et en numératie, les données indiquent que le lien entre le niveau d'instruction et les compétences en littératie dépend également d'autres facteurs (c.-à-d. éducation continue, expérience professionnelle, motivation, changements cognitifs) qui contribuent à l'acquisition, au maintien et à la perte des compétences en littératie au cours de la $v^{2}{ }^{51}$. En fait, le niveau d'instruction en tant que mesure de remplacement des compétences des adultes en littératie peut entraîner une erreur majeure ${ }^{51}$.

Environ 76 \% des participants ont indiqué qu'ils subiraient un test de dépistage du cancer colorectal s'ils connaissaient leur risque de développer cette maladie au cours de leur vie, Cependant, des statistiques récentes indiquent que le taux de tests de dépistage du cancer colorectal chez les adultes canadiens âgés de 50 ans et plus est inférieur à $15 \%{ }^{21}$. Tout en reconnaissant l'incohérence entre le nombre réel de tests de dépistage du cancer colorectal et le nombre de personnes ayant l'intention de subir un test de dépistage, le nombre d'adultes âgés exprimant leur intention de subir un dépistage préventif après avoir lu de l'information sur le risque lié au cancer colorectal était prometteur. En revanche, il est préoccupant de constater que $30 \%$ des participants n'ont pu donner des exemples de membres de la famille du premier degré à partir de la liste fournie dans l'information sur le cancer et la génétique, et que ce fait a été associé à un niveau insuffisant de littératie fonctionnelle en matière de santé. En effet, cette constatation prend une importance d'autant plus grande dans le contexte des recommandations actuelles concernant le dépistage chez tous les membres de la famille du premier degré des personnes ayant des marqueurs génétiques connus du cancer colorectal ${ }^{21}$.

De plus, le fait de se sentir personnellement concerné par l'information accroît la réflexion sur le contenu et aide à comprendre l'information grâce à l'attention accrue portée à celle-ci ${ }^{48}$. Les participants à l'étude n'avaient apparemment pas été confrontés directement au risque de développer le cancer colorectal et, par conséquent, ils étaient peut-être moins enclins à prêter attention aux messages de sensibilisation à cette maladie.

\section{Limites}

La présente étude comporte certaines limites. Le niveau de compréhension du risque chez les personnes âgées vivant d'une manière autonome dans la collectivité est peut-être différent de celui des personnes qui sont malades. En effet, la maladie peut 
influer sur les aptitudes cognitives liées au raisonnement et à la prise de décisions, altérant la capacité de la personne de bien comprendre l'information sur le risque ${ }^{52}$. De la même manière, les niveaux de littératie des participants ne sont pas représentatifs des Canadiens plus jeunes. Cependant, le risque plus élevé de cancer chez les adultes âgés a guidé le plan de l'étude. Par conséquent, les résultats actuels rendent compte d'un groupe de personnes âgées en bonne santé plutôt que d'un groupe de personnes malades.

Il faut tenir compte du biais de sélection potentiel découlant du fait que les personnes plus scolarisées sont plus susceptibles de se porter volontaires pour participer à une étude sur la compréhension de l'information sur la santé. Les personnes qui ne fréquentaient pas les bibliothèques ou les centres communautaires pour personnes âgées ne sont pas vraiment représentées dans l'étude. L'utilisation d'un échantillon de commodité tiré de ces établissements peut avoir découragé les adultes âgés ne disposant pas d'un moyen de transport (p. ex., ceux qui ne peuvent conduire, ceux qui sont handicapés physiquement). Cependant, divers statuts socioéconomiques étaient représentés, ce qui a permis d'obtenir des données transversales sur les personnes âgées. Par conséquent, cet échantillon de commodité rendait compte d'un groupe d'adultes âgés actifs, relativement mobiles.

Le fait que les propriétés des indices de la numératie en général et en matière de santé en particulier n'aient pas fait l'objet d'une validation psychométrique en dépit de l'utilisation courante de ces indices par les chercheurs dans le domaine 9,10,53-55 $^{\text {consti- }}$ tue une autre limite. Les analyses préliminaires révèlent une fiabilité suffisante ${ }^{9}$ des instruments d'évaluation utilisés tant pour la numératie en général qu'en matière de santé. La numératie en matière de santé peut être décrite comme un «domaine en évolution $»^{16}$. Ainsi, les trois mesures d'évaluation de la numératie utilisées dans la présente étude représentaient les instruments qui étaient disponibles au moment de la conception de l'étude, et elles ont été utilisées en vue d'assurer une évaluation

exhaustive des compétences des participants en numératie.

Les caractéristiques des patients ${ }^{42}$, de même que les modalités de présentation 31,43,44 influent sur la capacité de comprendre le risque. Les auteurs ont délibérément circonscrit leur étude sur la compréhension du risque de cancer dans les domaines de la littératie et de la numératie en matière de santé. La sélection des variables explicatives avait expressément pour but de combler les lacunes dans les recherches sur la numératie en matière de santé dans le contexte de la compréhension du risque.

Enfin, la capacité de lire l'information sur la santé diffusée sur Internet correspond aux niveaux de la $10^{\mathrm{e}}$ année et des $11^{\mathrm{e}}$ et $12^{\mathrm{e}}$ années. Ces niveaux de lecture étaient supérieurs aux niveaux de lecture de $5^{\mathrm{e}}$ ou de $6^{\mathrm{e}}$ année recommandés pour la population générale $^{56}$. Il s'agit d'une limite importante dans le cadre de l'étude de la compréhension de l'information. Cependant, les participants ont examiné l'information qu'ils auraient normalement consultée s'ils avaient effectué une recherche sur le site Web de la SCC de leur propre chef.

\section{Conclusion et incidence sur la pratique}

La présente étude est la première à examiner l'influence du niveau de littératie en matière de santé, et du niveau de numératie en particulier, sur la capacité des Canadiens âgés de comprendre l'information sur la prévention du cancer colorectal diffusée sur Internet. Les résultats de l'étude ont révélé que le niveau de numératie en matière de santé est un prédicteur constant de la capacité des participants de comprendre le risque à la lecture de l'information courante, de même que de l'information plus complexe, sur la prévention du cancer.

Le fait de constater que les niveaux de littératie et de numératie sont associés à une meilleure compréhension du risque chez les participants souligne la nécessité de clarifier les mots et les concepts dans l'information sur la santé diffusée sur Internet. Ainsi, on pourrait améliorer les messages de prévention impersonnels par la répétition de mots, de même que par l'explication des concepts moins courants, en vue d'aider le lecteur à comprendre l'information sur la prévention du cancer colorectal. Compte tenu du fait que même des opérations numériques de base peuvent être perçues comme étant plus complexes si elles s'inscrivent dans un contexte non familier, les spécialistes de l'information et les concepteurs de sites Web doivent relever le défi de concevoir de l'information Internet qui tient compte de divers niveaux de littératie en matière de santé.

On s'attend de plus en plus à ce que les personnes interviennent activement dans les décisions concernant leurs soins de santé. Il faut poursuivre les recherches en vue de préciser le concept de la numératie en matière de santé, d'élaborer des outils d'évaluation de la numératie exhaustifs, et d'examiner plus en détail le lien entre le niveau de littératie et de numératie en matière de santé dans tous les groupes d'âge, pour diverses maladies chroniques, et selon différents groupes ethniques.

\section{Remerciements}

L'étude a été réalisée grâce à une subvention des Instituts de recherche en santé du Canada. Les auteurs remercient notre partenaire communautaire, le Cancer Prevention and Early Detection Network of Waterloo Region, E. Harvey pour ses conseils concernant les analyses statistiques, de même que les 140 personnes qui ont participé à l'étude.

\section{Références}

1. Nielsen-Bohlman L, Panzer A, Kindig DA (dir.). Institute of Medicine (IOM). Health literacy: A prescription to end confusion, Washington, DC, The National Academies Press, 2004.

2. Agence de la santé publique du Canada (ASPC). Qu'est-ce qui détermine la santé? URL : http://www.phac-aspc.gc.ca/ph-sp/ determinants/index-fra.php .

3. Baker DW, Gazmararian JA, Sudano J, Patterson M. The association between age and health literacy among elderly persons. J Gerontol B Psychol Sci Soc Sci. 2000;55: S368-S374. 
4. Baker DW, Gazmararian JA, Williams MV, Scott T, Parker RM, Green D, et al. Health literacy and use of outpatient physician services by Medicare managed care enrollees. J Gen Intern Med. 2004; 19:215-220.

5. Baker DW, Parker RM, Williams MV, Clark WS. Health literacy and the risk of hospital admission. J Gen Intern Med. 1998; 13:791-798.

6. Fuller R, Dudley N, Blacktop J. Risk communication and older peopleunderstanding of probability and risk information by medical inpatients aged 75 years and older. Age Ageing. 2001; 30:473-476.

7. Gazmararian JA, Baker DW, Williams MV, Parker RM, Scott TL, Green DC, et al. Health Literacy among medicare enrollees in a managed care organization. JAMA. 1999;281:545-551.

8. Santé Canada. Effets du niveau d'alphabétisme sur la santé des Canadiens et des Canadiennes, Ottawa, Santé Canada, 2003. URL : http://www.travelhealth.gc.ca/ph-sp/ literacy-alphabetisme/literacy-fra.php .

9. Lipkus IM, Samsa G, Rimer BK. General performance on a numeracy scale among highly educated samples. Med Decis Making. 2001;21:37-44.

10. Schwartz L, Woloshin S, Black W, Welch H. The role of numeracy in understanding the benefit of screening mammography. Ann Intern Med. 1997;127: 966-972.

11. Schwartz SR, McDowell J, Yueh B. Numeracy and the shortcomings of utility assessment in head and neck cancer patients. Head Neck.. 2004;26:401-407.

12. Sheridan S, Pignone M, Lewis C. A randomized comparison of patients' understanding of number needed to treat and other common risk reduction formats. J Gen Intern Med.. 2003;18:884-892.
13. Williams MV, Parker RM, Baker DW, Parikh NS, Pitkin K, Coates WC, et al. Inadequate functional health literacy among patients at two public hospitals. JAMA. 1995;274: 1677-1682.

14. Wolf MS, Gazmararian JA, Baker DW. Health literacy and functional health status among older adults. Arch Intern Med. 2006;165:1946-1952.

15. Nurss J, Parker RM, Baker DW. TOFHLA: Test of Functional Health Literacy for Adults, Peppercorn Books and Press Inc, 1995.

16. Golbeck AL, Ahlers-Schmidt CR, Paschal AM, Dismuke SE. A definition and operational framework for health numeracy. Am J Prev Med. 2005;29:375-376.

17. Davids SL, Schapira M, McAuliffe TL, Nattinger AB. Predictors of pessimistic breast cancer risk perceptions in a primary care population. J Gen Intern Med. 2004; 19:310-315.

18. Sheridan SL, Pignone M. Numeracy and the medical student's ability to interpret data. Eff Clin Pract. 2002;5:35-40.

19. Woloshin S, Schwartz LM, Moncur M, Gabriel S, Tosteson AN. Assessing values for health: numeracy matters. Med Decis Making. 2001;21:382-390.

20. Weinstein ND, Atwood KA, Puleo E, Fletcher R, Colditz GA,Emmons K. Colon cancer: Risk perceptions and risk communication. J Health Commun. 2004;9: 53-65.

21. Société canadienne du cancer. Statistiques canadiennes sur le cancer, 2006. URL : http://www.cancer.ca .

22. Statistique Canada. Ménages qui utilisent Internet à la maison, selon le but de l'utilisation, Gouvernement du Canada, 2004. URL : http://www40.statcan.ca/102/cst01/ arts52b_f.htm .

23. Eysenbach G. Towards the millennium of cybermedicine. J Med Internet Res. 1999: 1;:S1,e2.
24. Charles C, Gafni A, Whelan T. Shared decision-making in the medical encounter: What does it mean? (or it takes least two to tango). Soc Sci Med. 1997;44:681-692.

25. Charles C, Whelan T, Gafni A. What do we mean by partnership in making decisions about treatment? BMJ. 1999;319:780-782.

26. Weinstein ND. What Does It Mean to Understand a Risk? Evaluating risk comprehension. J Natl Cancer Inst Monogr. 1999; 25:15-20

27. Ashcraft MH, Kirk EP. The relationship between working memory, math anxiety, and performance. J Exp Psychol Learn Mem Cogn. 2001;27:157-175.

28. Speros C. Health Literacy: concept analysis. J Adv Nurs. 2004;50:633-640.

29. Wilson-Fisher J. The crucial link between literacy and health. Ann Intern Med. 2003;139:875-878.

30. Riche JM, Reid JC, Robinson RD, Kardash $\mathrm{CM}$. Text and reader characteristics affecting the readability of patient literature. Read Improvement. 1991;28:287-292.

31. Kreuter MW. Dealing With competing and conflicting risks in cancer communication. J Natl Cancer Inst Monogr. 1999;25:27-35.

32. Jones CJ. CBA's that work. Assessing student's math content-reading levels. Teach Exceptional Child. 2001;34:24-28.

33. Baker DW, Williams MV, Parker RM, Gazmararian JA, Nurss J. Development of a brief test to measure functional health literacy. Patient Educ Couns. 1999;38:33-42.

34. Hopko DR, Mahadevan R, Bare RL, Hunt MK. The Abbreviated Math Anxiety Scale (AMAS): Construction, validity, and reliability. Assessment. 2003;10:178-182.

35. Schwartz LM, Woloshin S, Welch HG. Can patients interpret health information? An assessment of the medical data interpretation test. Med Dec Making. 2005;25:290-300. 
36. Hopko DR. Confirmatory factor analysis of the Math Anxiety Rating Scale-Revised. Educ Psychol Meas. 2003;63:336-351.

37. McLaughlin GH. SMOG grading - a new readability formula. J Read. 1969;12: 639-649.

38. Boyd JH, Watkins AR, Price CL, Fleming F, DeBaun MR. Inadequate community knowledge about sickle cell disease among African-American women. J Natl Med Assoc. 2005;9:62-67.

39. Richards M. Lay and professional knowledge of genetics and inheritance. Public Underst Sci. 1996;5;217-230.

40. Lanine AD, Jayaratne TE, Sheldon JP, Kardia SLR, Anderson ES, Feldbaum M, et al. Exploring the public understanding of basic genetic concepts. J Genet Couns. 2004; 13:305-320.

41. Donelle L, Arocha JF, Hoffman-Goetz L. Assessing health numeracy among community dwelling older adults. J Health Commun. 2007;12: 651-665.

42. Peters E, McCaul KD, Stefanek M, Nelson W. A heuristics approach to understanding cancer risk perception: contributions from judgment and decision-making research. Ann Behav Med. 2006;31:45-52.

43. Schapira $M M$, Nattinger $A B$, McHorney CA. Frequency or probability? A qualitative study of risk communication formats used in health care. Med Dec Making. 2001; 21:459-467.

44. Reid JC, Kardash CM, Robinson RD. Comprehension in patient literature: the importance of text and reader characteristics. Health Commun. 1994;6:327-335.

45. Ahlers-Schmidt CR, Golbeck AL, Paschal AM, Zackula R, Taylor NT. Breast cancer counts: numeracy in breast cancer information on the web. J Cancer Educ. 2006;21: 95-98.
46. Hirsch ED. Rea ding comprehension requires knowledge of the words and the world, 2003. URL : http://www.aft.org/ pubs-reports/american_educator/spring2003/ AE_SPRNG.pdf .

47. Beier ME, Ackerman PL. Age, ability, and the role of prior knowledge on the acquisition of new domain knowledge: promising results in a real-world learning environment. Psychol Aging. 2005;20:341-355.

48. Spires HA, Donley J. Prior knowledge activation: inducing engagement with informal texts. J Educ Psychol. 1998; 90:249-260.

49. Friedman, DB, Hoffman-Goetz L. An exploratory study of older adults' comprehension of printed cancer information: Is readability a key factor? J Health Commun. 2007;12:423-437.

50. Statistique Canada. Apprentissage et réussite : Premiers résultats de l'Enquête sur la littératie et les compétences des adultes, Ottawa et Paris, Organization for Economic Co-operation and Development, 2005. URL: http://www.statcan.ca/francais/ freepub/89-603-XIF/89-603-XIF2005001.htm .

51. Statistique Canada. Miser sur nos compétences : résultats canadiens de l'enquête internationale de l'alphabétisation et les compétences des adultes, Ottawa, Ressources humaines et Développement des compétences Canada, 2005, nº 89-617XIE au catalogue.

52. Cassell EJ, Leon AC, Daufman SG. Preliminary evidence of impaired thinking in sick patients. Ann Intern Med. 2001; 134:1120-1123.

53. Woloshin, S, Schwartz LM, Black WC, Welch HG. Women's perceptions of breast cancer risk: how you ask matters. Med Dec Making. 1999;19: 221-229.

54. Schapira M.M, Davids SL, McAuliffe TL, Nattinger AB. Agreement between scales in the measurement of breast cancer risk perceptions. Risk Anal. 2004;24: 665-673.
55. Estrada C, Martin-Hryniewicz M, BarnesHiggs V, Collins C, Byrd JC. Anticoagulant patient information material is written at high readability levels. Stroke. 2000; 31:2966-2970.

56. Estey A, Musseau A, Keehn L. Comprehension levels of patients reading health information. Patient Educ Couns. 1991;18: 165-169. 